\title{
El café y el desarrollo Histórico-Geográfico de Costa Rica: Vida y obra historiográfica de Carolyn Hall en perspectiva económica
}

Por: Br. José David Ramírez Roldán', Universidad de Costa Rica, Costa Rica

Recibido: $\quad 8$ de enero, 2019.

Aceptado: $\quad 2$ de abril, 2019.

\section{Resumen}

Si se habla de la renovación historiográfica desde mediados de los años setenta, es de importancia resaltar la investigación de la geógrafa inglesa Carolyn Hall sobre el ámbito de la geografía histórica costarricense. Por eso, el presente artículo reseña la vida académica de Carolyn Hall y sus contribuciones metodológicas a la historia de la historiografía costarricense, dándole atención a su obra principal El café y el desarrollo Histórico-Geográfico de Costa Rica. Asimismo, la investigación se adentra en el análisis de su influencia teórica para dar cuenta del aspecto económico de su trabajo.

\section{El café y el desarrollo Histórico-Geográfico de Costa Rica: The life and his-} toriographic works of Carolyn Hall from an economic point of view

\section{Abstract \\ When talking about historiographic renewal of the mid-70s, one would be remiss not to highlight British researcher Carolyn Hall's work in the field of Costa Rican historical geography. This study surveys Carolyn Hall's academic life and her con- tributions to methodology in the field of Costa Rican historiography history, paying special attention to her masterwork, El café y el desarrollo Histórico-Geográfico de Costa Rica. This research delves into an analysis of her theoretical influence to account for the economic aspect of her work.}

José David Ramírez Roldán. El café y el desarrollo Histórico-Geográfico de Costa Rica: Vida y obra historiográfica de Carolyn Hill en perspectiva económica. Revista Comunicación. Año 40, volumen 28, número 1, enero-junio, 2019. Instituto Tecnológico de Costa Rica. ISSN: 0379-3974 / e-ISSN1659-3820.
PALABRAS CLAVE:

Teoría económica, Historiografía, Café, Geografía histórica, Enseñanza superior.

KEY WORDS:

Economic theory, Historiography, Coffee, Historical geography, Higher education.
1 José David Ramírez es Bachiller en Historia, graduado de la Universidad de Costa Rica. Labora como docente en esa misma casa de estudios. Contacto: jdr_r@hotmail.com. 
Desde hace cuatro décadas atrás, la existencia de un cambio trascendental en la historiografía de Costa Rica se ha ido ofreciendo por medio de la consolidación científica y de constante profesionalización para las diversas áreas en estudio. Dicha proyección tomó recursos como "...programas de investigación, publicaciones y encuentros académicos..." (Arias, 2010). En otros términos, lo específico de la interpretación histórica es notable en la subjetividad y el posicionamiento de la narrativa científica del pasado y su intérprete, los cuales se alimentan siempre de una constante discusión mediática, ya sea entre los entendidos u otros públicos ajenos a la Academia.

Al iniciar la década de los años setenta, investigadores, en su mayoría extranjeros, como Carolyn Hall, Roger Churnside, Lowell Gudmunson y Jeffrey Casey, aportaron valiosos estudios científicos sobre el tema de la economía y la sociedad a la Academia de Historia Costarricense de aquel momento, gracias a importantes encuentros entre las contrapartes de sus fuentes primarias recabadas y consultadas, especialmente en periódicos y Memorias de Hacienda (Fischel, 1997, p. 49). Además, sus trabajos fueron vistos como incursiones pioneras y renovadas en un análisis metodológico interdisciplinario para logar una adecuada profesionalización de la historia en Costa Rica, para estudiar el período comprendido entre 1880 y 1930. En menor grado, se ofreció un agregado informativo de índole económico y social, por los historiadores Carlos Araya, Mario Oliva y Vladimir de la Cruz (ídem, pp. 4950).

Estas interpretaciones de Hall han sido valiosas como marco contextual para el estudio de los fenómenos políticos e ideológicos; así ha sido señalado por Fischel (1997). En ese mismo contexto, la influencia conjunta de los profesores de historia y geografía: Hall, Cardoso, Pérez Brignoli, Tjarks, Gould, Araya Pochet y Paulino González Ilegaron a transformar con profundidad las temáticas de investigación, gracias a la introducción de novedosos enfoques en los programas del estudio histórico en la década de 1970 (Solórzano Fonseca, 2013, p. 29).

Al hablar de aquella renovación historiográfica dada a mediados de los años setenta, es de suma importancia, resaltar la excelente investigación de la geógrafa inglesa Carolyn Hall sobre el ámbito de la geografía histórica costarricense. Su trabajo se tituló El café y el desarrollo histórico geográfico de Costa Rica, y fue defendido originalmente como tesis doctoral en Oxford para 1972; posteriormente, la escritora se mudó a Costa Rica durante la misma década, y su famosa obra fue pública en 1976, en formato de libro. Empero, a pesar de los años que tiene la obra, sigue siendo un clásico de la literatura del desarrollo económico cafetalero para nuestro país. En efecto, esto ya lo había señalado casi de manera profética la doctora Esther Jimeno: "este trabajo....se va a convertir para los estudiantes universitarios en un "modelo" por su sólida estructura, rigor metodológico, claridad expositiva y profundidad investigaba" (1976).

Afirmaciones como la anterior, se leen y escuchan repetidamente en las bocas y manos de diversos especialistas que se han interesado por la historia de la caficultura costarricense o, como describiría Samper Kutschbach, "...el mejor libro que se ha publicado sobre el tema...". Por consiguiente, voy a proponer entonces que hay una relación posible, entre la revolución historiográfica costarricense y su acercamiento a la aplicabilidad de las teorías económicas. Me ocuparé, para ello, de un caso específico, al estudiar este libro, basado en la tesis doctoral de la profesora Hall; pero no pretendo y no es mi intención, hacer una mancuerna simbiótica con toda la historia de la historiografía costarricense.

Más bien, el presente artículo rescata el aporte historiográfico al campo de la historia económica y eco-agraria costarricense, ofrecido por el texto $\mathrm{El}$ café y el desarrollo histórico geográfico de Costa Rica de Carolyn Hall. Para ello, se realiza principalmente un análisis metodológico desde la teoría económica que presenta la obra. Además, se muestran algunos aspectos de las representaciones formadas en la opinión académica sobre la profesora Hall.

\section{VIAJE Y TRAYECTORIA INTELECTUAL DE UNA EXTRANJERA}

En esta sección, se presenta consideradamente el aporte intelectual de la Dra. Carolyn Hall "(...) por su trayectoria como investigadora y como 
promotora de la geografía histórica en la región centroamericana..." (Arteaga, 1982, p. 198) y especialmente, en Costa Rica. Además, considero interesante referenciar su labor como mentora y maestra, a la luz de las facetas personales que sean pertinentes para el estudio de ese vínculo.

Samper Kutschbach (2007) ha expresado que "Carolyn Hall fue la arquitecta principal de la Geografía Histórica -propiamente tal- en y sobre Costa Rica" (p. 9). Esta afirmación se respalda en el enfoque geográfico británico presente en las investigaciones de la profesora Hall, el cual daba un uso aplicado a las fuentes y procedimientos de la ciencia histórica y los mezclaba con herramientas de la Geografía. En fin, el objetivo era revolucionar el estudio de los "procesos geográficos pretéritos y de pertinencia actual" (ibid).

Este tipo de geografía contextualizadora, se logró con medios meramente académicos, brindados por los cimientos intelectuales presentes en la Escuela de Historia y Geografía de la Universidad de Costa Rica; dicho diálogo interdisciplinario mezcló los aportes del abordaje francés e inglés y logró mejoras metodológicas y estructurales en las disciplinas de Geografía e Historia. Asimismo, hay que evidenciar la preparación metodológica dada a los investigadores costarricenses antes de la llegada de la profesora Hall, cuya formación en ambas disciplinas tuvo incursiones pioneras significativas en el quehacer académico de la geografía histórica, especialmente los influidos por la forma de ver y hacer la Historia en la Escuela de los Annales. De hecho, se dio "primero por intermediación española y luego a través de destacados investigadores radicados en el país al mismo tiempo que la Dra. Hall -caso de Ciro Cardoso y Héctor Pérez- o que hicieron sus posgrados en el exterior durante esos años" (ibid). Pero antes de todos estos aportes que revolucionaron la forma de ver el espacio-tiempo del pasado costarricense, hubo estudiosos, tanto nacionales como extranjeros que, de igual forma, procuraron realizar investigaciones preliminares que relacionaran una perspectiva en conjunto de la historia y la geografía. Entre los más importantes se destacaron, Carlos Meléndez (1926-2000) y Gerhard Sandner (1929-2013).
Podríamos irnos más atrás de esa unión entre historia y geografía para continuar hablando sobre la historiografía nacional, pero la profesora Hall ha dejado muy en claro el problema de las obras decimonónicas y en su mayoría, también las anteriores a los años setenta, al expresar que, "durante la última década la investigación en las ciencias sociales ha florecido, como nunca antes en la historia intelectual de Costa Rica. Han proliferado tesis y artículos, informes y monografías. Sin embargo, las obras de interpretación y síntesis son contadas" (Clare, 2011). Hay que anotar que este trabajo mencionado es, "...una obra precursora de lo que ha dado en Ilamarse historia ambiental..." (Samper Kutschbach, 2007, p. 10) y analizar esa relación entre ambiente y sociedad. El defecto que evidencia la Dra. Hall presente en las obras decimonónicas y anteriores a la revolución intelectual de la ciencias sociales en Costa Rica, no es, ni más ni menos que, el sesgo de un abordaje patrio, tradicionalista y positivista que, más allá de querer hacer una síntesis narrativa y descriptiva, "...pretendía fundamentar una república naciente" (ibid, p. 9).

Carolyn Hall tuvo en cada obra y proyecto investigativo la misma rigurosidad metódica en el cotejo de las fuentes históricas y su aplicabilidad, y las relacionó con las herramientas geográficas. Puede notarse esta firmeza desde su tesis doctoral hasta sus artículos de 1974 y 1975 (Samper Kutschbach, 2007, p. 10).

Además, entre 1976 y 1983, fue pionera en muchos otros campos de estudio, por ejemplo, abordó la historia empresarial desde la investigación histórico-geográfica del proceso agro del país. Para ello, confróntense sus textos de 1976 y 1978 (Samper 2007, p. 10).

Todos sus trabajos ofrecen un destacado enfoque, modulado para crear una excelente perspectiva económica de la geografía regional costarricense, pero uno de sus trabajos con mayor relevancia actual lo brinda al estudiar las disparidades territoriales, en su artículo, "Regional Inequalities in Well-Being in Costa Rica", de 1984. Durante las décadas de los ochenta y noventa, interpretó el territorio nacional con temáticas como las evoluciones económicas de los ecosistemas indígenas, la herencia colonial, los 
cambios paisajísticos, las disparidades regionales de la distribución de la riqueza y el ordenamiento territorial en Costa Rica, vistos "...desde las cuestiones físico-geográficas y ambientales... (Samper Kutschbach, 2007). Además, esta investigadora siempre buscó estudiar el campo interdisciplinario entre la Historia y la Geografía (Hall, 1989).

La Dra. Hall tuvo también aportes medulares que marcarían el estudio centroamericano y proyectarían el ámbito histórico-geográfico costarricense a escala ístmica. Pero su contribución más importante fue haberse dedicado a producir durante más de una década, el Atlas Histórico de Centroamérica (Hall, Pérez y Cotter, 2003). Su versión original fue publicada en inglés.

Es necesario resaltar que los historiadores costarricenses, gracias a los medios públicos formales, no vieron de mala gana la intervención y contribución intelectual a la geografía e historia de Costa Rica y del istmo centroamericano. Todo lo contrario, posiblemente, acostumbrados a recibir aportes foráneos como academia y en un contexto más abierto a distintos posicionamientos científicos, la década de los setentas marco el inicio de una "...Geografía Histórica moderna, con solidez conceptual y metodológica" (Samper, 2007). Así:

como figura fundacional y preeminente... a esta británica que llegó como joven estudiante de posgrado a hacer su trabajo de campo, se enamoró del país...y se quedó hasta la fecha, aportando al medio académico nacional, con sus potencialidades y sus limitaciones, la solidez de una Geografía pensada históricamente, y contribuyó asimismo a sus diálogos con una Historia que tomaba mayor conciencia de su dimensión geográfica (Samper Kutschbach , p. 10).

Finalmente, Carolyn Hall siempre fue exigente como profesora que, muy en consonancia con la rigidez de sus trabajos, se adoptó de forma "... desinteresada en la popularidad fácil, e incapaz de claudicar, pero igualmente empeñada en mostrar a sus estudiantes y colegas que era posible y necesario, siempre, mejorar" (Samper Kutschbach, 2007, p. 9), como lo señala su compañero de labores, Mario Samper, quien también apreciaba su "...calidez humana que se expresa más fácilmente fuera del salón de clase o de reunión, en su hospitalaria acogida, en la charla informal y en los momentos difíciles de la vida" (ibid), mientras se constituía diversos trabajos académicos editoriales a su nombre. Ahora bien, estamos frente a una colectividad ístmica adscrita a los aportes científicos de la Dra. Hall, donde se construía una relación epistemológica y metodológica “...entre Geografía e Historia, sobre el objeto de estudio y la naturaleza de la Geografía Histórica como campo" (ibid, p. 9), ofreciéndose a sí misma, una inmortalidad como personaje intelectual en la historia de la historiografía centroamericana.

\section{ANÁLISIS TEÓRICO-METODOLÓGICO DE LA ECONOMÍA EN HALL}

El libro El café y el desarrollo Histórico-Geográfica de Costa Rica, como ya se ha señalado bastante, dio el primer gran acercamiento al significado del cultivo del café en nuestro país. Antes de la década de los setenta, la evolución de la industria cafetalera no había sido estudiada de forma científica; el enfoque novedoso y la nueva metodología utilizada en la obra de la Dra. Hall, fue particularmente revolucionario en Costa Rica, y resultó la piedra angular de un renovado marco de estudio para el campo histórico-geográfico, el cual modernizó con métodos disciplinarios traídos de su alma mater, la Universidad de Oxford.

Al inicio de la obra, su autora, deja en claro, en los siguiente términos, la búsqueda "...de la localización e interrelaciones de los diferentes fenómenos relevantes al tema de la industria cafetalera...así como de examinar la contribución de esa industria a los procesos de cambio geográfico de Costa Rica a través del tiempo" (Arteaga, 1982). Por ende, durante toda su obra, se aprecia una profunda inmersión en documentos históricos, estadísticos y asentamientos agrícolas y se destaca la búsqueda de fuentes primarias encontradas en la Biblioteca Nacional de Costa Rica o archivos públicos y privados (por ejemplo: el Archivo General de las Indias, el Archivo Nacional de Costa Rica y los archivos de los hacendados en Costa Rica), con lo que logró 
emplear censos estadísticos, protocolos coloniales, registro de la propiedad, revistas y periódicos.

La Dra. Hall, no se aparta del esquema tradicional de la teoría de la dependencia y el análisis macroeconómico, criterios muy en boga de aquellos estudiosos del campo económico durante los años sesenta y setenta, quienes querían explicar el subdesarrollo en los países latinoamericanos. En su obra, se tratan términos como "renta per cápita" y "consumo" y se estudia la mortalidad infantil desde el campo democrático. Empero, matiza esta teoría económica con un criterio más contextualizador, donde su metodología moderna "...toma en cuenta las estructuras básicas y la evolución histórica de los fenómenos que caracterizan al tercer mundo" (ibid).

Su análisis de la historia agro-económica con respecto a la aparición del grano de oro y su arraigo en la sociedad costarricense, posee una influencia estructuralista que revela distintos datos interesantes; por ejemplo, resultan indudables las mejores condiciones sociales de los campesinos propietarios en Costa Rica, comparadas con las de los trabajadores de las grandes haciendas guatemaltecas o salvadoreñas, donde la estructura material condiciona lo social, las relaciones económicas mutan como evidencia empírica en el ámbito de lo social (Ibid, p. 200).

Por otro lado, la autora logra demostrar una sobresaliente relación en el desarrollo de la economía cafetalera y las instituciones, este punto de vista institucionalista nos acerca a comprender el papel del proceso evolutivo de las instituciones sociales para la formación del comportamiento económico del mercado caficultor; por ejemplo, dentro de estas medidas estatales, aparece en 1969 la publicación del "Primer Plan Nacional de Política Cafetalera", asimismo, se toma en cuenta intentos de regular del mercado exportador, por medio de la creación del Instituto de Defensa del Café, institución que posteriormente pasó a llamarse la Oficina del Café. De hecho, "esta dependencia tomó medidas para reglamentar los pagos hechos por el grano dentro de Costa Rica y también para proteger los precios y mercados de los exportadores" (ibid, p. 199).

\section{REFLEXIÓN}

Con el deseo de profundizar aún más en el tema, me dediqué a la tarea de buscar información complementaria en otras fuentes bibliográficas, con la sorpresa de encontrarme en un libro llamado Café, sociedad y relaciones de poder en América Latina, editado por William Roseberr y Lowell Gudmundson, una llamativa nota al pie en el capítulo IV escrita por Mario Samper, Ilamado "Tiempos Difíciles: Los caficultores colombianos y costarricense entre la prosperidad y la crisis, 1920-19362 (Samper Kutschbach, 2001). Así, en una de las notas aclaratorias, se nos comunica a los lectores que la tesis de la Dra. Hall analiza parcialmente los datos del censo cafetalero de 1935, ya que se llega a omitir curiosamente la información sobre cafetos por finca. Paralelamente, se señala que la tesis del profesor Rodrigo Facio (1941), fue tomada en cuenta por Hall, pero también, Ilega a dejar de lado el estudio minucioso de Facio dentro de su reproducción y comentarios de datos presentados el año anterior por el director del Instituto de Defensa del Café (Roseberry y Gudmundson, 2001, p. 261).

En fin, la obra dibuja en la mente del lector con trazos finos y cuidadosos un recorrido histórico de la Costa Rica de inicios del siglo XIX hasta 1971, y más allá del ejercicio narrativo, "analiza con mucho detalle la infraestructura económica de la industria del café y su evolución, así como la colonización agrícola en y fuera del Valle Central, para concluir con un análisis del "Café y la Crisis del Subdesarrollo entre 1934/71" (Arteaga, 1982, p. 261).

\section{REFERENCIAS BIBLIOGRÁFICAS:}

Arias, D. (2010). Historiografía sin camisas de fuerza, o las prácticas del comentar: en torno al libro de Isabel Gamboa Barboza, En el Hospital Psiquiátrico. El sexo como (lo)cura. Istmo Revista virtual de estudios literarios y culturales centroamericanos, (21), 1-25. Disponible en: https://www.academia.edu/9397537/_2010_ Historiograf\%C3\%ADa_sin_camisas_de_ fuerza_o_las_pr\%C3\%A1cticas_del_comentar en_torno_al_libro_de_Isabel_Gamboa_Barboza_ En_el_Hospital_Psiqui\%C3\%A1trico._El_sexo_ como_lo_cura_Istmo_jul-dic_2010. 
Arteaga, R. (1982). El café y el desarrollo históricogeográfico de Costa Rica de Carolyn Hall. Revista de la integración y el desarrollo de Centroamérica del Banco Centroamericano de Integración Económica, (40), 198-201.

Clare, P. (2011). Reflexiones para una historia ambiental de Costa Rica relevante y pertinente. Del Proyecto Agua, Tierra, Aire y Bosque: historia y medio ambiente en Costa Rica (Siglos XIX-XXI), San José: CIHAC.

Facio, R. (1941). Estudio sobre economía costarricense. (Tesis de licenciatura en Derecho). Universidad de Costa Rica, Costa Rica.

Fischel, A. (1997). Historiografía y Educación en Costa Rica: un aporte. En Martínez, A. y Narodowski, M. (Comp.), Escuela, historia y poder (pp. 37-54). Buenos Aires: Ediciones Novedades Educativas.

Hall, C. (1989). La geografía histórica: un campo interdisciplinario entre la geografía y la historia. En Fonseca, E. (Comp.), Historia. Teoría y métodos (pp. 42). San José: EDUCA.

Hall, C. (1984). Regional Inequalities in Well-Being in Costa Rica. Geographical Review, 74, 1, 48-62.

Hall, C. (1976). Los archivos de Keith: algunos aspectos de la geografía histórica de Costa Rica. 1871-1873. Revista de Costa Rica, 11, 101-127

Hall, C. (1981). Costa Rica una interpretación geográfica con perspectiva histórica. San José: Editorial Costa Rica y Universidad Nacional.

Hall, C. (1976). El café y el desarrollo históricogeográfico de Costa Rica. San José: Editorial Costa Rica.

Hall, C. (1975). La expansión de los transportes en Costa rica. Revista Geográfica de América Central (Costa Rica), (2), 3, 9-26.

Hall, C. (1974). La jerarquía urbana de Costa Rica: una consideración de la aplicación de modelos geográficos. Revista Geográfica de América Central (Costa Rica), 1, 25-48.

Hall, C. (1978). The Tuis Archives: Cattle Ranching on The Frontiers of Colonization in Costa Rica. 1873-1876. Revista Geográfica (Instituto Panamericano de Geografía e Historia), 86-87, 101-117.

Hall, C. (1978). Cóncavas: formación de una hacienda cafetalera, 1889-1911. San José: Editorial Universidad de Costa Rica.

Hall, C., Pérez, H. y Cotter, J. (2003), Historical Atlas of Central America. Norman: University of Oklahoma Press.

Jimeno, E. (1976). Carolyn Hall: El café y el desarrollo histórico-geográfico de Costa Rica. Revista Geográfica de América Central, 1 (4), 108- 111.

Martínez, A. y Narodowski, M. (1997). Escuela, historia y poder: miradas desde América Latina. Buenos Aires: Ediciones Novedades Educativas.

Roseberry, W. y Gudmundson, L. (2001). Café, sociedad y relaciones de poder en América Latina. San José: EUNA.

Samper Kutschbach, M. (2007). Carolyn Hall y el desarrollo de la geografía Histórica en Costa Rica. Revista Historia, 55-56, 9-12.

Samper Kutschbach, M. (2001). Tiempos difíciles: los caficultores colombianos y costarricenses entre la prosperidad y la crisis, 1920-1936. En Samper, M. Roseberry, W. y Gudmundson, L. Café, sociedad y relaciones de poder en América Latina, (eds.) (pp. 243-310). Heredia: EUNA.

Solórzano Fonseca, J. C. (2013). Los años cuando el Dr. Ciro Cardoso fue profesor de la Escuela de Historia y Geografía de la Universidad de Costa Rica: impacto en sus discípulos. Revista de Historia, 68, 27-39. 